\title{
FUNCTIONAL DIFFERENTIAL EQUATIONS WITH INFINITE DELAY IN BANACH SPACES
}

\author{
JIN LIANG \\ Teaching and Research Section of Mathematics, \\ Kunming Engineering Institute, Kunming, P.R. China \\ TIJUN XIAO \\ Department of Mathematics, Yunnan Teachers' University, \\ Kunming, P.R. China \\ (Received August 17, 1989 and in revised form August 16, 1990)
}

\begin{abstract}
In this paper, a definition of the fundamental operator for the linear autonomous functional differential equation with infinite delay in a Banach space is given, and some sufficient and necessary conditions of the fundamental operator being exponentially stable in abstract phase spaces which satisfy some suitable hypotheses are obtained. Moreover, we discuss the relation between the exponential asymptotic stability of the zero solution of nonlinear functional differential equation with infinite delay in a Banach space and the exponential stability of the solution semigroup of the corresponding linear equation, and find that the exponential stability problem of the zero solution for the nonlinear equation can be discussed only in the exponentially fading memory phase space.
\end{abstract}

KEY WORDS AND PHRASES: Functional differential equation with infinite delay, Banach space, fundamental operator, exponentially stable, phase space.

1980 Mathematics Subject Classification (1985 Revision). Primary 34K20, 34D20.

\section{INTRODUCTION}

We consider the functional differential equation with delay

$$
\dot{x}=f\left(t, x_{t}\right),
$$

where $x_{t}(\theta)=x(t+\theta),-\infty<\theta \leq 0$, and $x$ takes values in a Banach space $E$. Because many phenomena in nature which vary in time can be written in the form of (1.1), the study of (1.1) has been a significant and interesting subject. Since the 70 s, the theory of the functional differential equation with delay has been developed swiftly, a lot of important results have been obtained (see [1]). However, as stated in [1], most of the papers dealing with this subject require that $E$ be a finite-dimensional space. Therefore, the case when $E$ is an infinite-dimensional space must be researched further.

The main motivation for this paper was a desire to take a step in this direction. We investigated the exponential stability problem for linear or nonlinear functional differential equations with infinite delay when $E$ is an infinite-dimensional space. A definition of the fundamental operator for the linear autonomous functional differential equation with infinite delay in a Banach space is given in Section 2, which si the generalization of the fundamental matrix (see [1, § 3]). In Section 3 , we overcame successfully the difficulty caused by infiniteness of dimension of the space, and obtained some sufficient and necessary conditions for the fundamental operator to be exponentially stable in an abstract phase space which satisfy some 
suitable hypotheses. Some examples of the phase spaces are given in Section 4. In Section 5, we discussed the relation between the exponential asymptotic stability of the zero solution of a nonlinear functional differential equation with infinite delay in a Banach space and the exponential stability of the solution semigroup of the corresponding linear equation, and found that the exponential stability problem for the nonlinear equations can be discussed only in the exponentially fading memory phase spaces.

\section{THE DEFINITION AND PROPERTIES OF THE FUNDAMENTAL OPERATOR}

Let $E$ be a Banach space, denote by $\|\cdot\|$ a norm in $E$, and by $R, R_{+}, R_{-}$the real, the non-negative real, the non-positive real numbers, respectively. Let $B$ be a linear vector space of functions mapping $(-\infty, 0]$ into $E$, and assume that $B$ is a Banach space with the norm $\|\cdot\|_{B}$ and satisfies the following hypotheses:

(H1) If $b x:(-\infty, A] \rightarrow E, A>\sigma$, is continuous on $[\sigma, A]$ and $x_{\sigma} \in B$, then $x_{t} \in B$ and $x_{t}$ is continuous in $t \in[\sigma, A]$.

(H2) $\|x(0)\| \leq K\|x\|_{B}$ for all $x$ in $B$ and some constant $K$.

(H3) There exist a continuous function $K(t)$ of $t \geq 0$, and a function $M(t)$ which is non-negative, locally bounded on $[0, \infty)$ and submultiplicative, that is, $M(t+s) \leq M(t) M(s)$ for $t, s \geq 0$, such that

$$
\left\|x_{t}\right\|_{B} \leq K(t-\sigma) \sup \{\|x(s)\| ; \sigma \leq s \leq t\}+M(t-\sigma)\left\|x_{\sigma}\right\|_{B}
$$

for $\sigma \leq t \leq A$ and $x$ having the properties in (H1).

or $(\mathrm{H} 1)(\mathrm{H} 2)$ and

(H4) Let $x, y \in B$, and $\|x(\theta)\| \leq\|y(\theta)\|$ for a.e. $\theta \in R_{-}$, then there exists a constant $M_{1}$ such that $\|x(\cdot)\|_{B} \leq M_{1}\|y(\cdot)\|_{B}$.

We consider the linear autonomous functional differential equation

$$
\begin{cases}\dot{x}(t)=L\left(x_{t}\right), & t>0 \\ x(\theta)=\phi(\theta), & \theta \in R_{-},\end{cases}
$$

where $L$ is a linear bounded operator mapping $B$ into $E, \phi \in B$.

It is easy to verify that for each $\phi \in B$, the solution of $(2.1) x(t)=x(t, \phi)$ exists uniquely for $t \in R_{+}$. Moreover, by (H1), the solution operators $T(t), t \geq 0$, defined by

$$
(T(t) \phi)(\theta)=x_{t}(\theta)=x_{t}(\theta, \phi) \text { for } \phi \in B,
$$

is strongly continuous semigroup of bounded linear operators on $B$. If $L=0$, we denote the solution semigroup by $S(t)$.

By the arguments similar to the Theorem 4.4 in [8], we have

LEMMA 2.1. There exists a real $\omega_{1}$ such that $\exp (\lambda \cdot) b \in B$ for $b \in E$ and $R e \lambda>\omega_{1}$, and it is an analytical function of $\lambda$.

PROOF. Let

$$
(C(t) \phi)(\theta)= \begin{cases}\exp (-(t+\theta)) \phi(0), & t+\theta \geq 0 \\ \phi(t+\theta), & t+\theta<0,\end{cases}
$$

where $\phi(\theta) \in B$. Then $C(t)$ is a $C_{0}$-semigroup in $B$. If $\mathcal{A}(\mathcal{B})$ is the infinitesimal generator of $C(t)(S(t))$, by semigroup theory [9], there is $\omega_{1} \geq 0$ such that for $\operatorname{Re} \lambda>\omega_{1}$,

$$
\begin{gathered}
(R(\lambda ; \mathcal{A}) \phi)(\theta)-(R(\lambda ; \mathcal{B}) \phi)(\theta) \\
=\int_{0}^{\infty} \exp (-\lambda t)(C(t) \phi)(\theta) d t-\int_{0}^{\infty} \exp (-\lambda t)(S(t) \phi)(\theta) d t
\end{gathered}
$$




$$
\begin{aligned}
& =\int_{0}^{\infty} \exp (-\lambda t)[(C(t) \phi)(\theta)-(S(t) \phi)(\theta)] d t \\
& =\int_{-\theta}^{\infty} \exp (-\lambda t)(\exp (-(t+\theta)) I-I) \phi(0) d t \\
& =-\frac{1}{\lambda(\lambda+1)} \exp (\lambda \theta) \phi(0) .
\end{aligned}
$$

Since (H1) implies that the set $\{\phi(0) ; \phi \in B\}$ coincides with $E$, we obtain Lemma 2.1 .

LEMMA 2.2. There exists a constant $\omega \geq \omega_{1}$ such that $(\lambda I-L(\exp (\lambda \cdot)))^{-1}$ exists for $\operatorname{Re} \lambda>\omega$, where $L(\exp (\lambda \cdot))$ is a linear operator from $E$ to $E$ defined by $L(\exp (\lambda \cdot)) b=L(\exp (\lambda \cdot) b)$ for $b \in E$.

PROOF. If (H1) (H2) (H3) hold, by (H3) we have that there exists $\omega_{0} \geq \omega_{1}$, such that $\|\exp (\lambda \cdot) b\|_{B}(b \in E)$ is uniformly bounded for $\operatorname{Re} \lambda>\omega_{0}$. So, by the boundedness of $L$ and the BanachSteinhaus theorem, we get that $L(\exp (\lambda \cdot))$ is uniformly bounded for $\operatorname{Re} \lambda>\omega_{0}$. If follows that there is a sufficiently large $\omega \geq \omega_{0}$ such that $(\lambda I-L(\exp (\lambda \cdot)))^{-1}$ exists for $R e \lambda>\omega$. If $(\mathrm{H} 1)(\mathrm{H} 2)(\mathrm{H} 4)$ hold, by $(\mathrm{H} 4)$ we have

$$
\| L\left(\exp (\lambda \cdot) b\|\leq\| L\|\| \exp (\lambda \cdot) b\left\|_{B} \leq L\right\| M_{1}\left\|\exp \left(\omega_{0} \cdot\right) b\right\|_{B}\right.
$$

for $\operatorname{Re} \lambda \geq \omega_{0}$ and $b \in E$, where $\omega_{0}>\omega_{1}$. According to the same reason as above, we obtain the conclusion.

Set $\Delta(\lambda)=\lambda I-L(\exp (\lambda \cdot))$. From Lemma 2.1 and Lemma 2.2, $\Delta(\lambda)$ is an analytic function of $\lambda$ for $\operatorname{Re} \lambda>\omega_{1}$, and $\Delta^{-1}(\lambda)=(\lambda-L(\exp (\lambda \cdot)))^{-1}$ exists for $\operatorname{Re} \lambda>\omega$.

Define

$$
X(t)=\left\{\begin{array}{c}
\int_{c)} \exp (\lambda t) \Delta^{-1}(\lambda) d \lambda=\operatorname{Lim}_{T \rightarrow \infty} \int_{c-i T}^{c+i T} \frac{1}{2 \pi i} \exp (\lambda t) \Delta^{-1}(\lambda) d \lambda,(t>0), \\
I,(t=0) ; 0,(t<0)
\end{array}\right.
$$

where $\operatorname{Re} c>\omega$, we call this operator-value function the fundamental operator of (2.1).

Using the arguments similar to those in [8], we obtain:

THEOREM 2.3. Let $(\mathrm{H} 1)-(\mathrm{H} 3)$ or $(\mathrm{H} 1)(\mathrm{H} 2)(\mathrm{H} 4)$ hold, $X(t)$ be the fundamental operator of $(2.1)$. Then

i) There exists real $\mu>\omega$, such that

$$
\Delta^{-1}(\lambda)=\int_{0}^{\infty} \exp (-\lambda t) X(t) d t \text { for } \operatorname{Re} \lambda \geq \mu .
$$

ii) $X(t)$ is a continuous function of $t$ in $[0, \infty)$.

iii) For any $\varepsilon>0$, there exists a $C(\varepsilon)$ such that

$$
\|X(t)\| \leq C(\varepsilon) \exp (\mu+\varepsilon) t \text { for } t \geq 0
$$

\section{THE EXPONENTIAL STABILITY OF THE FUNDAMENTAL OPERATOR}

The fundamental operator $X(t)$ is said to exponentially stable if there exist positive numbers $G$ and $r$, such that $\|X(t)\| \leq G \exp (-r t)$ for $t \geq 0$.

Denote by $\chi_{[u, v]}(\cdot)$ the characteristic function of $[u, v]$.

$$
\chi_{[-,, 0]}(\cdot) b \in B \text { for any } t>0 \text { and } b \in E \text {. }
$$

$$
L\left(\chi_{[-,, 0]}[\cdot) b\right) \text { is well defined for any } t>0 \text { and } b \in E \text {. }
$$


(H6)

$$
\text { The operator-value function } L\left(\chi_{t-1,0\}}(\cdot)\right) \text { mapping } t \in R_{+} \text {into } B(E, E) \text {, defined by }
$$

$$
L\left(\chi_{\{-1,0]}(\cdot)\right) b=L\left(\chi_{[-1,0]}(\cdot) b\right) \text { for } b \in E,
$$

is left continuous, where $B(E, E)$ stands for the space of all bounded linear operators form $E$ to $E$.

LEMMA 3.1. Let $B$ satisfy (H1) (H2) (H3) (H5), and (H6) hold. Then

i) For $\operatorname{Re} \lambda>\omega(\omega$ the constant in Lemma 2.2$)$,

$$
L\left(\int_{0}^{\infty} \exp (-\lambda t) \chi_{[-1,0]}(\cdot) d t\right)=\frac{1}{\lambda} L(\exp (\lambda \cdot))=\int_{0}^{\infty} \exp (-\lambda t) L\left(\chi_{\{-1,0]}(\cdot)\right) d t .
$$

ii) The fundamental operator of (2.1) satisfies the equation

$$
\begin{cases}\dot{X}(t)=L\left(X_{t}\right), & t>0, \\ X(t)=0(t<0), & X(0+)=1 .\end{cases}
$$

PROOF. By Lemma 2.2, $L(\exp (\lambda \cdot) b)$ is well defined for $\operatorname{Re} \lambda>\omega$ and $b \in E$, and $L(\exp (\lambda \cdot) b)=L(\exp (\lambda \cdot)) b$. Thus

$$
L\left(\int_{0}^{\infty} \exp (-\lambda t) \chi_{[-1,0]}(\cdot) d t\right)=\frac{1}{\lambda} L(\exp (\lambda \cdot))
$$

From (H5), $L\left(\exp (-\lambda t) \chi_{[-r, 0]}(\cdot) b\right)$ is well defined for $t>0$ and $b \in E$, and is equal to $\exp (-\lambda t) L\left(\chi_{[-, 0)}(\cdot)\right) b$. By the continuity of $L$ and (H6), we obtain

$$
L\left(\int_{0}^{\infty} \exp (-\lambda t) \chi_{[-1,0]}(\cdot) d t\right)=\int_{0}^{\infty} \exp (-\lambda t) L\left(\chi_{[-1,0]}(\cdot)\right) d t,
$$

so i) holds.

Set $Y_{t}(\cdot) b=X_{t}(\cdot) b-\chi_{\{-, 0\}}(\cdot) b$ for $b \in E$, then by $(\mathrm{H} 1), Y_{t}(\cdot) b \in B$. Thus, by $(\mathrm{H} 5), L\left(X_{t}(\cdot) b\right)$ is well defined for $t>0$ and $b \in E$. Thereby, by virtue of $\mathrm{i})$ and the definition of the fundamental operator of (2.1), for any $b \in E$, taking the Laplace inverse transforms of the two sides of $\Delta^{-1}(\lambda) b=\frac{1}{\lambda} b+\frac{1}{\lambda} L(\exp (\lambda \cdot)) \Delta^{-1}(\lambda) b$, we have

$$
X(t) b=b+\int_{-\theta}^{t} L(X(s+\theta) b) d s=b+\int_{0}^{t} L\left(X_{s}\right) b d s \text { for } t>0,
$$

where $\theta \in R_{-}$. It implies $X(t)$ is the solution of (3.1), so ii) holds.

REMARK. ii) of Lemma 3.1 implies that the fundamental operator is the generalization of the fundamental matrix defined by (40) and (41) in [1].

THEOREM 3.2. Let $B$ satisfy (H1)-(H3) (H5), (H6) hold, $L(\exp (\lambda \cdot))$ be analytic for $R e \lambda>0$ and continuous for $\operatorname{Re} \lambda \geq 0, \Delta^{-1}(\lambda)$ exist for $\operatorname{Re} \lambda>0$ and there exist positive numbers $W, Q, N$ and $M$ such that $|K(t)| \leq W,|M(t)| \leq Q\left(K(t), M(t)\right.$ is the functions in (H3), $\left.t \in R_{+}\right),\left\|X_{0}\right\| \leq N$ and $\|L(\exp (\lambda \cdot))\| \leq M$. Then the fundamental operator of $(2.1)$ is exponentially stable if and only if there is a positive constant $\varepsilon$, such that:

i) $\Delta^{-1}(\lambda)$ can be extended analytically to the half plane $\operatorname{Re} \lambda>-\varepsilon$,

ii) $\operatorname{Lim}_{|\omega| \rightarrow \infty} \sup _{\nu_{1} \leq \nu \leq v_{2}}\left\|\Delta^{-1}(\nu+i \omega)\right\|=0$ for $v_{1}, v_{2} \in(-\varepsilon, \infty)$,

iii) For any $f \in E^{*}$ (the dual space of $E$ ), $b \in E$ and $v>-\varepsilon$, there is a constant $J$ such that

$$
\int_{-\infty}^{\infty}\left|f\left(\Delta^{-1}(v+i \omega) b\right)\right|^{2} d \omega \leq \frac{J}{v+\varepsilon}\|f\|^{2}\|b\|^{2} .
$$

In order to prove this theorem, we shall use the following well known result. 
LEMMA 3.3 [5, P. 409] Suppose that $\int_{-\infty}^{\infty}|f(x)| d x<\infty$ and $\sup _{x \in R}\{f(x)\}<\infty$, and that the Fourier transform $\tilde{f}(y)$ is real-valued and non-negative. Then $\int_{-\infty}^{\infty} \tilde{f}(y) d y \leq 2 \pi \sup _{x \in R}\{f(x)\}$

The proof of Theorem 3.2. Necessity. In view of Theorem 2.3, there exists a real constant $\mu$, such that

$$
\Delta^{-1}\left(\lambda=\int_{0}^{\infty} \exp (-\lambda t) X(t) d t \text { for } \operatorname{Re} \lambda>\mu .\right.
$$

Suppose $X(t)$ is exponentially stable, that is, there exist positive constants $G$ and $r$ such that $\|X(t)\| \leq G \exp (-r t)$ for $t \geq 0$. Then for $R e \lambda>-r$, the integral on the right side of (3.2) converges absolutely and defines an analytic function of $\lambda$ in the half plane $R e \lambda>-r$. It is just the analytic extension of $\Delta^{-1}(\lambda)$ to the half plane $\operatorname{Re} \lambda>-r$, that is, i) holds.

For any $-r<v_{1}<v<v_{2}<\infty$,

$$
\begin{aligned}
\Delta^{-1}(v+i \omega) & =\int_{0}^{\infty} \exp (-v t) X(t) \exp (-i \omega t) d t \\
& =-\int_{0}^{\infty} \exp (-v t) X(t) \exp \left(-i\left(t+\frac{\pi}{\omega}\right) \omega\right) d t \\
& =-\int_{\frac{\omega}{\omega}}^{\infty} \exp \left(-v\left(t-\frac{\pi}{\omega}\right)\right) X\left(t-\frac{\pi}{\omega}\right) \exp (-i \omega t) d t
\end{aligned}
$$

Hence,

which implies that ii) holds.

$$
\begin{aligned}
\left\|\Delta^{-1}(v+i \omega)\right\| & \leq \frac{1}{2}\left\|\int_{0}^{\infty}\left[\exp (-v t) X(t)-\exp \left(-v\left(t-\frac{\pi}{\omega}\right)\right) X\left(t-\frac{\pi}{\omega}\right)\right] \exp (-i \omega t) d t\right\| \\
& +\frac{1}{2}\left\|\int_{0}^{\frac{\pi}{\omega}} \exp \left(-v\left(t-\frac{\pi}{\omega}\right)\right) X\left(t-\frac{\pi}{\omega}\right) \exp (-i \omega t) d t\right\|,
\end{aligned}
$$

Let $\lambda=v+i \omega, v>-\gamma$. From (3.2), for any $f \in E^{*}$ and $b \in E$, we obtain

$$
\begin{aligned}
\left|f\left(\Delta^{-1}(v+i \omega) b\right)\right|^{2} & =\left(\int_{0}^{\infty} \exp (-(v+i \omega) t) f(X(t) b) d t, \int_{0}^{\infty} \exp (-(v+i \omega) s) f(X(s) b) d s\right)_{1} \\
& =\int_{0}^{\infty} \int_{0}^{\infty} \exp (-v(t+s)) \exp (-i \omega(t-s))(f(X(t) b), f(X(s) b))_{1} d s d t \\
& =\int_{0}^{\infty} \int_{-s}^{\infty} \exp (-i \omega u) \exp (-v(u+2 s))(f(X(s+u) b), f(X(s) b))_{1} d u d s \\
& =\int_{-\infty}^{\infty} \exp (-i \omega u) F(u) d u,
\end{aligned}
$$

where $(\cdot, \cdot)_{1}$ stands for the inner product of the complex plane,

$$
F(u)=\int_{0}^{\infty} \exp (-v(u+2 s))(f(X(s+u) b), f(X(s) b))_{1} d s \text { as } u \geq 0,
$$


and

$$
F(u)=\int_{-u}^{\infty} \exp (-v(u+2 s))(f(X(s+u) b), f(X(s) b))_{1} d s \text { as } u<0 .
$$

For $u \geq 0$,

$$
\begin{aligned}
|F(u)| & \left.\leq \int_{0}^{\infty} G^{2} \exp (-v(u+2 s)) \exp (-r(u+s)) \exp -r s\right)\|f\|^{2}\|b\|^{2} d s \\
& =[2(v+r)]^{-1} G^{2} \exp (-(v+r) u)\|f\|^{2}\|b\|^{2} .
\end{aligned}
$$

Similarly, for $u<0$, we have

$$
|F(u)| \leq[2(v+r)]^{-1} G^{2} \exp ((v+r) u)\|f\|^{2}\|b\|^{2} .
$$

Therefore, set $\varepsilon=r, J=\frac{\sigma}{2}$, by virtue of Lemma 3.3 we see that iii) holds.

SUFFICIENCY. By i) and ii), we get that

$$
X(t)=\frac{1}{2 \pi i} \operatorname{Lim}_{T \rightarrow \infty} \int_{c-i T}^{c+i T} \exp (\lambda t) \Delta^{-1}(\lambda) d \lambda
$$

is independent of $c$, for $c>-\varepsilon$.

From the assumptions of this Theorem, we deduce that there is a constant $D$ such that

$$
\left\|\Delta^{-1}(\lambda)\right\| \leq \frac{D}{1+|\lambda|} \text { for } \operatorname{Re} \geq 0 \text {. }
$$

By (3.3),

$$
\begin{aligned}
X(t) & =\frac{1}{2 \pi i} \operatorname{Lim}_{T \rightarrow \infty} \int_{-T}^{i T} \exp (\lambda t) \Delta^{-1}(\lambda) d \lambda=\frac{1}{2 \pi} \operatorname{Lim}_{T \rightarrow \infty} \int_{-T}^{T} \exp (i \omega t) \Delta^{-1}(i \omega) d \omega \\
& =\frac{1}{2 \pi} \int_{-T_{0}}^{T_{0}} \exp (i \omega t) \Delta^{-1}(i \omega) d \omega+\frac{1}{2 \pi} \operatorname{Lim}_{T \rightarrow \infty}\left[\int_{-T}^{-T_{0}}+\int_{T_{0}}^{T}\right] \frac{\exp (i \omega t)}{i \omega}\left[I+L(\exp (i \omega \cdot)) \Delta^{-1}(i \omega)\right] d \omega .
\end{aligned}
$$

where $T_{0} \geq 0$ is a constant. Since $\Delta^{-1}(i \omega)$ is continuous and bounded on $\left[-T_{0}, T_{0}\right], \|(i \omega)^{-1} L(\exp (i \omega \cdot)) \Delta^{-1}$ $(i \omega) \|$ is integrable on $\left[T_{0}, \infty\right)$ and $\left(-\infty,-T_{0}\right]$ by means of $\|L(\exp (i \omega \cdot))\| \leq M$ and (3.4), making use of the arguments similar to those in the proof of ii) of the necessity of this Theorem, and noting that (integrating by parts)

$$
\operatorname{Lim}_{t \rightarrow \infty} \operatorname{Lim}_{T \rightarrow \infty}\left[\int_{-T}^{-T_{0}}+\int_{T_{0}}^{T}\right] \frac{\exp (i \omega t)}{i \omega} d \omega=0,
$$

we obtain $\operatorname{Lim}_{t \rightarrow \infty}\|X(t)\|=0$. Hence, there is a constant $D_{0}>0$ such that $\|X(t)\| \leq D_{0}$. Thus, by virtue of Lemma 3.1, (H3), $|K(t)| \leq W,|M(t)| \leq Q$, and $\left\|X_{0}\right\| \leq N$, we have

$$
\|\dot{X}(t)\|=\left\|L\left(X_{t}(\cdot)\right)\right\| \leq W\|L\| D_{0}+M\|L\| N \triangleq D_{1}
$$

Suppose $0<p<\varepsilon, \lambda=-p+i \omega, b \in E, f \in E^{*}$, then by (3.3),

$$
\exp (p t) f(X(t) b)=\frac{1}{2 \pi} \operatorname{Lim}_{T \rightarrow \infty} \int_{-T}^{T} \exp (i \omega t) f\left(\Delta^{-1}(-p+i \omega) b\right) d \omega .
$$

Therefore, thanks to Plancherel formula [10] and iii), we obtain

$$
\begin{aligned}
\int_{0}^{\infty} \exp (2 p t)|f(X(t) b)|^{2} d t & =\frac{1}{2 \pi} \int_{-\infty}^{\infty}\left|f\left(\Delta^{-1}(-p+i \omega) b\right)\right|^{2} d \omega \\
& \leq J(2 \pi(\varepsilon-p))^{-1}\|f\|^{2}\|b\|^{2} .
\end{aligned}
$$

Taking $t_{0}=0$, then there is $t_{1}>t_{0}$ with $t_{1}-t_{0} \leq 1$ such that $\left|f\left(X\left(t_{1}\right) b\right)\right|^{2} \leq \exp \left(-2 p t_{1}\right) J(\pi(\varepsilon-p))^{-1}\|f\|^{2}\|b\|^{2}$. 
In fact, if not, that is, for any $t \in\left[t_{0}, t_{0}+1\right],|f(X(t) b)|^{2}>\exp (-2 p t) V(\pi(\varepsilon-p))^{-1}\|f\|^{2}\|b\|^{2}$, thus,

$$
\int_{0}^{\infty} \exp (2 p t)|f(X(t) b)|^{2} d t \geq \int_{i_{0}}^{t_{0}+1} \exp (2 p t)|f(X(t) b)|^{2} d t \geq J(\pi(\varepsilon-p))^{-1}\|f\|^{2}\|b\|^{2},
$$

which is in contradiction with (3.6). So, we can choose a strictly increasing number sequence $\left\{t_{m}\right\}_{1}^{\infty}$ with $t_{0}=0$ and $t_{m+1}-t_{m} \leq 1$ such that

$$
\left|f\left(X\left(t_{m}\right) b\right)\right|^{2} \leq J \exp \left(-2 p t_{m}\right)(\pi(\varepsilon-p))^{-1}\|f\|^{2}\|b\|^{2}, m=1,2, \ldots
$$

Since

$$
\frac{d}{d t}|f(X(t) b)|^{2}=2 \operatorname{Re}(f(\dot{X}(t) b), f(X(t) b))_{1},
$$

it follows that

$$
|f(X(t) b)|^{2}=\left|f\left(X\left(t_{m}\right) b\right)\right|^{2}+2 R e \int_{t_{m}}^{t}(f(\dot{X}(\tau) b), f(X(\tau) b))_{1} d \tau .
$$

For each $t \in R_{+}$, there is an $m$ such that $t \in\left[t_{m}, t_{m+1}\right]$. Hence, from (3.5), (3.7) and (3.8), we obtain

$$
\begin{aligned}
|f(X(t) b)|^{2} & \leq J \exp \left(-2 p t_{m}\right)(\pi(\varepsilon-p))^{-1}\|f\|^{2}\|b\|^{2} \\
& +2 D_{1}\|f\|\|b\| \int_{t_{m}}^{t}|f(X(\tau) b)| d \tau \\
& \leq J \exp (2 p) \exp (-2 p t)(\pi(\varepsilon-p))^{-1}\|f\|^{2}\|b\|^{2} \\
& +2 D_{1}\left\{\int_{t_{m}}^{t} \exp (-2 p \tau) d \tau\right\}^{\frac{1}{2}}\left\{\int_{t_{m}}^{t} \exp (2 p \tau) \mid f\left(\left.x(\tau) b\right|^{2} d \tau\right\}^{\frac{1}{2}}\|f\|\|b\|\right. \\
& \leq J \exp (2 p) \exp (-2 p t)(\pi(\varepsilon-p))^{-1}\|f\|^{2}\|b\|^{2} \\
& +\frac{\sqrt{J} D_{1}}{\sqrt{\pi p(\varepsilon-p)}} \exp \left(-p t_{m}\right)\|f\|^{2}\|b\|^{2} \\
& \leq\left\{\frac{J}{\pi(\varepsilon-p)} \exp (2 p) \exp (-2 p t)\right. \\
& \left.+\exp (p) \exp (-p t) \frac{\sqrt{J} D_{1}}{\sqrt{\pi p(\varepsilon-p)}}\right\}\|f\|^{2}\|b\|^{2} .
\end{aligned}
$$

Let $G=\left\{\frac{J_{\operatorname{epp}(p)}}{\pi(\varepsilon-p)}+\frac{\sqrt{J D_{1}}}{\sqrt{\pi p(\varepsilon-p)}}\right\}^{\frac{1}{2}} \exp \left(\frac{P}{2}\right)$. Then

$$
|f(X(t) b)| \leq G \exp \left(-\frac{p}{2} t\right)\|f\|\|b\|
$$

and consequently,

$$
\|X(t)\| \leq G \exp \left(-\frac{p}{2} t\right),(t \geq 0) .
$$

This ends the proof of this Theorem.

REMARK 1. If $E$ is a Hilbert space, then the iii) in Theorem 3.2 can be changed into the following iii') There exists a constant $J$ such that

$$
\int_{-\infty}^{\infty}\left\|\Delta^{-1}(v+i \omega)\right\|^{2} d \omega \leq \frac{J}{v+\varepsilon} \text { for } v>-\varepsilon
$$


PROOF. Sufficiency is obvious. Noting that

$$
\left\|\Delta^{-1}(v+i \omega)\right\|^{2}=\left(\int_{0}^{\infty} \exp (-(v+i \omega) t) X(t) d t, \int_{0}^{\infty} \exp (-(v+i \omega) s) X(s) d s\right)
$$

where $(\cdot, \cdot)$ stands for the inner product of $E$, by the arguments similar to those in the proof of the necessity of Theorem 3.2, we can prove the necessity.

REMARK 2. It is clear that if we substitute (H3) with (H4) in Lemma 3.1, the conclusions of this lemma are also true, and if we substitute $(\mathrm{H} 4)$ and $\left\|\chi_{[-, 0\}}(\cdot) b\right\|_{B} \leq N\|b\|(N$ is a constant, $b \in E, t>0)$ for (H3), $|K(t)| \leq W,|M(t)| \leq Q$ and $\left\|X_{0}\right\| \leq N$ in Theorem 3.2, the conclusion of this theorem is true also.

THEOREM 3.4. Let $B$ satisfy (H1)-(H3), $\tilde{B}$ a linear vector space $(\tilde{B} \supset B), L$ a linear continuous operator from $\tilde{B}$ to $E,\left(\mathrm{H} 5^{\prime}\right)$ and $(\mathrm{H} 6)$ hold. Let $L(\exp (\lambda \cdot))$ be analytic for $\operatorname{Re} \lambda>0$ and continuous for $\operatorname{Re} \lambda \geq 0, \Delta^{-1}(\lambda)$ exist for $\operatorname{Re} \lambda \geq 0$ and there exists $M$ such that $\|L(\exp (\lambda \cdot))\| \leq M$. Suppose $X(t)$ is the fundamental operator of equation $(\phi(\theta) \in B)$

$$
\begin{cases}\dot{x}(t)=\left.L\right|_{B}\left(x_{t}\right), & t>0, \\ x(\theta)=\phi(\theta), & \theta \in R_{-},\end{cases}
$$

and $\left\|L\left(X_{t}\right)\right\| \leq C \sup _{t \geq 0}\|X(t)\|$, where $C$ is a constant. Then the conclusions of Lemma 3.1 and Theorem 3.2 hold.

The proof is similar to the proof of Lemma 3.1 and Theorem 3.2, so, we omit it.

According to the arguments similar to those in the proof [8, Proposition 6.4], we can obtain the following

THEOREM 3.5 Let $(\mathrm{H} 1)-(\mathrm{H} 3)$ or $(\mathrm{H} 1)(\mathrm{H} 2)(\mathrm{H} 4)$ hold for $B$, there exist positive constant $C$ and $r$ such that $L(\exp (\lambda \cdot))$ can be extended analytically to the half plane $\operatorname{Re} \lambda>-r$, and $\|L(\exp (\lambda \cdot))\| \leq C$ for any $\operatorname{Re} \lambda>-r$. Then the fundamental operator of (2.1) is exponentially stable if there exists a positive number $r_{1} \in(0, r)$ such that $\Delta^{-1}(\lambda)$ exists in the half plane $\operatorname{Re} \lambda \geq-r_{1}$.

\section{EXAMPLES OF PHASE SPACES}

If the space $B$ is defined as in Section 2, i.e., it satisfies (H1), (H2), (H3) or (H1), (H2), (H4), then we call $B$ a phase space. In this section, we shall give some examples of phase spaces and the operators which are satisfying (H5), (H6) or (H5'), (H6).

EXAMPLE 1. Let $B=\left\{\phi(\theta) ; \phi(\theta)\right.$ is a measurable function on $R_{-}$with $\int_{R_{-}} \exp (r \theta)\|\phi(\theta)\|^{P} d \theta<\infty$,

where $r>0$ and $p \geq 1$, with the norm

$$
\|\phi\|_{B}=\left\{\int_{-\infty}^{0} \exp (r \theta)\|\phi(\theta)\|^{P} d \theta\right\}^{\frac{1}{p}}+\|\phi(0)\| .
$$

It is easy to verify that $B$ is a Banach space and satisfies (H1) (H2)-(H5). Suppose $L$ is a bounded linear operator from $B$ to $E$, we deduce easily that (H6) holds, the hypotheses of Theorem 3.2 and Theorem 3.5 are satisfied. By virtue of Theorem 3.5, we obtain that the fundamental operator of the corresponding linear autonomous functional differential equation is exponentially stable if there exists a positive number $r_{1} \in(0, r)$ such that $\Delta^{-1}(\lambda)$ exists for $R e \lambda>-r_{1}$.

EXAMPLE 2. Let $B=\left\{\phi(\theta) ; \phi(\theta) \in C\left(R_{-}, E\right)\right.$ the space of all continuous functions from $R_{-}$to $E$, and $\operatorname{Lim} \exp (r \theta) \phi(\theta)=0, r>0\}$. with the norm

$$
\|\phi\|_{B}=\sup _{-\infty<\theta \leq 0} \exp (r \theta)\|\phi(\theta)\|
$$


Let $A_{t}$ be linear operators from $E$ to $E(i=1,2, \ldots), B(s): R_{+} \rightarrow B(E, E)$ is measurable, $t_{i} \geq 0(i=1,2, \ldots)$, and

$$
\sum_{i=1}^{\infty} \exp \left(r t_{t}\right)\left\|A_{\imath}\right\|<\infty \int_{0}^{\infty} \exp (s r)\|B(s)\| d s<\infty
$$

Set $L(x)=\sum_{i=1}^{\infty} A_{t} x\left(-t_{t}\right)+\int_{0}^{\infty} B(s) x(-s) d s$ for any $x(\cdot) \in B$.

Clearly, $B$ is a Banach space. From (4.1) it follows that $L$ is a linear bounded operator, $L$ and $B$ satisfy (H1)-(H4), (H5') and (H6). Furthermore, the corresponding fundamental operator satisfies

$$
\begin{cases}\dot{X}(t)=\sum_{i=1}^{\infty} A_{i} X\left(t-t_{i}\right)+\int_{0}^{t} B(s) X(t-s) d s, & t>0, \\ X(0+)=I, X(t)=0, & t<0 .\end{cases}
$$

Also by (4.1), we get that there is a constant $C$ such that $\left\|L\left(X_{t}\right)\right\| \leq C \sup \{\|X(t)\|, t \geq 0\}$. It is easy to verify that the hypotheses of Theorem 3.5 are satisfied. Hence, if there exists a positive number $r_{1} \in(0, r)$ such that $\Delta^{-1}(\lambda)$ exist for $R e \lambda>-r_{1}$, then $X(t)$ is exponentially stable.

EXAMPLE 3. Let $B=\left\{\phi(\theta) ; \phi(\theta)\right.$ is bounded uniformly continuous function from $R_{-}$to $\left.E\right\}$, with the norm $\|\phi(\cdot)\|_{B}=\sup _{\theta \in R_{-}}\|\phi(\theta)\|, L$ is the operator of Example 2 as $r=0$.

It is easy to prove that $L$ and $B$ satisfy the hypotheses of Theorem 3.4, therefore, $X(t)$ is exponentially stable if and only if there exists a positive number $\varepsilon$ such that i), ii) and iii) of Theorem 3.2 hold.

\section{THE NONLINEAR EQUATIONS IN BANACH SPACES}

We consider the nonlinear equation

$$
\begin{cases}\dot{x}(t)=f\left(t, x_{t}\right), & t>0 \\ x(t)=\phi(t), & t \in R_{-} .\end{cases}
$$

Let $f(t, \phi): R_{+} \times B \rightarrow E$ is continuous, and for each $\phi \in B$, the solution $x(t, \phi)$ of (5.1) exist uniquely for $t \in R_{+}$. Then

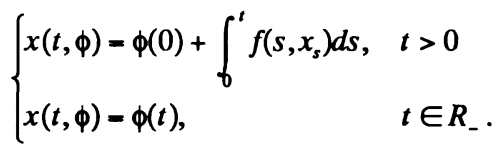

Denote by $U(t)$, defined by $(U(t) \phi)(\theta)=x_{t}(\theta, \phi)=x(t+\theta, \phi)$ for $t \in R_{+}$and $\theta \in R_{-}$, the solution mapping of (5.1). Let $f(t, 0)=0$. The solution $x=0$ of (5.1) is said to be exponentially asymptotically stable if there exist positive constants $M, \delta$ and $\alpha$ such that $\|\phi\|_{B}<\delta$ implies $\|U(t) \phi\|_{B} \leq M \exp (-\alpha t)$.

Set

$$
\left(\tau^{\prime} \phi\right)(\theta)=\left\{\begin{array}{c}
0,-t<\theta \leq 0 \\
\phi(t+\theta), \theta \leq-t
\end{array}, t \in R_{+}, \theta \in R_{-}, \phi \in B .\right.
$$

If there exist positive numbers $C$ and $r$ such that $\left\|\tau^{t} \phi\right\|_{t} \leq C \exp (-\alpha t)\|\phi\|_{B}$ for any $\phi \in B$ and $t>0$, then the phase space $B$ is called to be an exponentially fading memory space, where $\|\phi\|_{\imath}=\inf \left\{\|\psi\|_{B} ; \psi \in B\right.$ and $\psi(\theta)=\phi(\theta)$ for any $\theta \in(-\infty,-t]\}$.

THEOREM 5.1. Let $B$ be a phase space and the solution $x=0$ of (5.1) is exponentially asymptotically stable. Then $B$ is an exponentially fading memory space.

PROOF. Since $(U(t) \phi)(\theta)=\phi(t+\theta)=\left(\tau^{\prime} \phi\right)(\theta), \theta \leq-t$, for any $t>0$. Thus for any $\phi \in B$ and $\|\phi\|_{B} \leq \delta$, we have 


$$
\left\|\tau^{\prime} \phi\right\|_{l}=\|U(t) \phi\|_{t} \leq\|U(t) \phi\|_{B} \leq M \exp (-\alpha t) \quad(t \geq 0) .
$$

Hence, for any $\phi \in B,\left\|\tau^{\prime}\left(\|\phi\|_{B}^{-1} \delta \phi\right)\right\|_{s} \leq M \exp (-\alpha t)$. This fact implies

$$
\left\|\tau^{\prime} \phi\right\|_{t} \leq M \delta^{-1} \exp (-\alpha t)\|\phi\|_{B},
$$

that is, $B$ is a exponentially fading memory space.

Suppose $f\left(t, x_{t}\right)=L\left(x_{t}\right)+h\left(t, x_{t}\right)+g(t)$, where $L$ is a linear bounded operator from $B$ and $E, h(t, \phi): R_{+} \times B \rightarrow E$ is continuous and satisfies

$$
\|h(t, \phi)-h(t, \psi)\| \leq N\|\phi-\psi\|_{B} \text { for any } \phi, \psi \in B,
$$

where $N$ is a constant, $h(t, 0)=0, g(t): R_{+} \rightarrow E$ is continuous. Let $B$ satisfy (H1)-(H3) (H5) and (H6) hold. The linear equation corresponding to (5.1) is

$$
\left\{\begin{array}{l}
\dot{x}(t)=L\left(x_{t}\right), \quad t>0, \\
x(t)=\phi(t), \quad t \in R_{-} .
\end{array}\right.
$$

According to Lemma 3.1, the fundamental operator $X(t)$ of (5.3) is the solution of (3.1). Thereby, $X_{t}(\cdot)=T(t) X_{0}$, where $T(t)$ is the solution semigroup of (5.3) and

$$
X_{0}(\theta)=\left\{\begin{array}{l}
I, \theta=0 \\
0, \theta<0 .
\end{array}\right.
$$

The proofs of Proposition 6.1 and Theorem 6.5 in [8] tell us that

$$
(U(t) \phi)(\theta)=x_{t}(\theta, \phi)=(T(t) \phi)(\theta)+\int_{0}^{t+\theta} X(t+\theta-s)\left(h\left(s, x_{s}\right)+g(s)\right) d s, t+\theta \geq 0 .
$$

Since $X(t+\theta-s)=0$ as $s>t+\theta$,

$$
\begin{aligned}
(U(t) \phi)(\theta) & =(T(t) \phi)(\theta)+\int_{f}^{t} X_{t}(\cdot-s)\left(h\left(s, x_{s}\right)+g(s)\right) d s \\
& =(T(t) \phi)(\theta)+\int_{f}^{t} T(t-s) X_{0}\left(h\left(s, x_{s}\right)+g(s)\right) d s,
\end{aligned}
$$

i.e, we have

THEOREM 5.2. Let $f\left(t, x_{t}\right)=L\left(x_{t}\right)+h\left(t, x_{t}\right)+g(t), L$ be a linear bounded operator from $B$ to $E$, $h(t, \phi): R_{+} \times B \rightarrow E$ be continuous and satisfy $(5.2), h(t, 0)=0, g(t): R_{+} \rightarrow E$ be continuous. Let $B$ satisfy $(\mathrm{H} 1)-(\mathrm{H} 3)(\mathrm{H} 5)$ and $(\mathrm{H} 6)$ hold, and $x(0, \phi)(t)$ be the solution of $(5.1)$. Then

$$
\begin{aligned}
& x_{t}(0, \phi)(\theta)=(T(t) \phi)(\theta)+\int_{0}^{t} T(t-s) X_{0}\left(h\left(s, x_{s}\right)+g(s)\right) d s, t>0, \theta \in R_{-}, \\
& x(0, \phi)(\theta)=\phi(\theta), \theta \leq 0,
\end{aligned}
$$

where $T(t)$ is the solution semigroup of (5.3), $X_{0}=\left\{\begin{array}{l}I, \theta=0 \\ 0, \theta<0\end{array}\right.$.

REMARK. Theorem 5.2 gives actually a variation-of-constants formula.

THEOREM 5.3. Let $f\left(t, x_{t}\right)=L\left(x_{t}\right)+h\left(t, x_{t}\right), L$ be a linear bounded operator from $B$ to $E$, $h(t, \phi): R_{+} \times B \rightarrow E$ be continuous and satisfy $(5.2), h(t, 0)=0$. Let $B$ satisfy (H1)--(H3)(H5) and (H6) hold. Suppose the solution semigroup $T(t)$ of (5.2) is exponentially stable, that is, there exist $M, \delta>0$ such that $\|T(t)\| \leq M \exp (-\delta t)$. If $\left\|X_{0}\right\| \leq C(C$ a constant), and $C N M<\delta$, then the zero solution of (5.1) is exponentially asymptotically stable.

PROOF. Clearly, the solution of (5.1) exists uniquely. 
From Theorem 5.2, we have

$$
(U(t) \phi)(\theta)=(T(t) \phi)(\theta)+\int_{0}^{t} T(t-s) X_{0} h\left(s, x_{s}\right) d s,
$$

hence,

$$
\left.\|U(t) \phi\|_{B} s \| T(t) \phi\right)\left\|_{B}+\int_{f}^{t}\right\| T(t-s)\|\| h\left(s, x_{s}\right) \| d s .
$$

By (5.2) and $h(t, 0)=0$, we get $\|h(t, \phi)\| \leq N\|\phi\|_{B}$. Therefore,

$$
\|U(t) \phi\|_{B} \leq M \exp (-\delta t)\|\phi\|_{B}+C M N \int_{0}^{t} \exp (-\delta(t-s))\|U(s) \phi\|_{B} d s,
$$

that is,

$$
\exp (\delta t)\|U(t) \phi\|_{B} \leq M\|\phi\|_{B}+C N M \int_{0}^{t} \exp (\delta s)\|U(s) \phi\|_{B} d s .
$$

According to Bellmen inequality, we obtain

$$
\|U(t) \phi\|_{B} \leq M \exp (-\delta t)\|\phi\|_{B} \exp (C N M t) \text {. }
$$

So, for $\|\phi\|_{B} \leq \alpha,\|U(t) \phi\|_{B} \leq \alpha M \exp (-(\delta-C N M) t)$. This fact implies that the conclusion of the theorem holds.

THEOREM 5.4. Let $f\left(t, x_{t}\right)=L\left(x_{t}\right)+h\left(t, x_{t}\right), L$ be a linear bounded operator from $B$ to $E$, $h(t, \phi): R_{+} \times B \rightarrow E$ be continuous and satisfy $(5.2), h(t, 0)=0$. Let $B$ satisfy (H2)--(H3)(H5) and (H6) hold. Suppose the zero solution of (5.1) is exponentially asymptotically stable, that is, there exist $M, \delta, \alpha>0$ such that $\|\phi\|_{B} \leq \alpha$ implies $\|U(t) \phi\|_{B} \leq M \exp (-\delta t)$. If $\left\|X_{0}\right\| \leq C$ (C a constant), and $C N M<\alpha \delta$, then the solution semigroup of (5.3) is exponentially stable.

PROOF. By Theorem 5.2,

$$
(T(t) \phi)(\theta)=(U(t) \phi)(\theta)-\int_{0}^{t} T(t-s) X_{0} h\left(s, x_{s}\right) d s .
$$

So,

$$
\|T(t) \phi\|_{B} \leq U(t) \phi\left\|_{B}+\int_{0}^{t}\right\| T(t-s)\|\| X_{0}\|\| h\left(s, x_{s}\right) \| d s,
$$

hence, when $\|\phi\|_{B} \leq \alpha$, we have

$$
\|T(t) \phi\|_{B} \leq M \exp (-\delta t)+C N M \int_{0}^{t}\|T(t-s)\| \exp (-\delta s) d s .
$$

Since $T(t)$ is a linear operator, thus

$$
\|T(t)\|=\sum_{\|\psi\|_{B}-\alpha} \frac{\|T(t) \psi\|_{B}}{\|\psi\|_{B}}
$$

thereby,

$$
\exp (\delta t)\|T(t)\| \leq \frac{M}{\alpha}+\frac{C N M}{\alpha} \int_{0}^{t}\|T(s)\| \exp (\delta s) d s .
$$

According to Bellmen inequality, we obtain

$$
\|T(t)\| \leq \frac{M}{\alpha} \exp (-\delta t) \exp \left(\frac{C N M}{\alpha} t\right)=\frac{M}{\alpha} \exp \left(-\left(\delta-\frac{C N M}{\alpha}\right) t\right),
$$

thus, by $C N M<\alpha \delta$, the solution semigroup of (5.3) is exponentially stable and the proof is complete.

ACKNOWLEDGMENT. We are grateful to our advisor Professor F. L. Huang for his generous guidance. 


\section{REFERENCES}

1. Corduneanu, C. and Lakshmikantham, V., Equations With Unbounded Delay: A Survey, Nonlinear Analysis Theory Method and Application, 4 (1980), 831-877.

2. Corduneanu, C. and Luca, N., The Stability of Some Feedback Systems With Delay, J. Math. Anal. Appl. 51 (1975), 377-393.

3. Hale, J. K., Theory of Functional Differential Equations, Springer-Verlag, New York, 1977.

4. Hale, J. K. and Kato, J., Phase Space for Retarded Equations With Infinite Delay, Funci. Ekvaciaj, 21 (1978), 11-41.

5. Hewitt, E. and Stromberg, K., Real and Abstract Analysis, Springer-Verlag, New York, 1965.

6. Huang, F. L., On Linear Autonomous Functional Differential Equations With Infinite Delay, Ann. of Diff. Eqs. 3(3), 1987, 275-292.

7. -.----, On the Exponential Stability Problem for Linear Autonomous Functional Differential Equations with Infinite Delay, Scientia Sinica, Series A, 5 (1988). (Chinese)

8. Naito, T., On Linear Autonomous Retarded Equations With An Abstract Phase Space For Infinite Delay, J. Diff. Eqns. 33 (1979), 74-91.

9. Pazy, A., Semigroups of Linear Operators and Applications to Partial Differential Equations, (1983), Springer-Verlag, New York and Berlin.

10. Yosida, K. Functional Analysis, Springer-Verlag Berlin Heidelberg, 1965. 


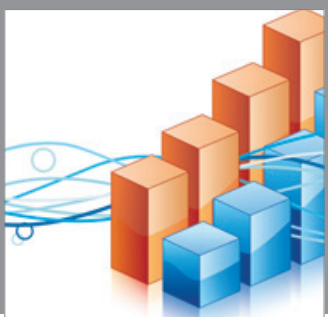

Advances in

Operations Research

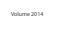

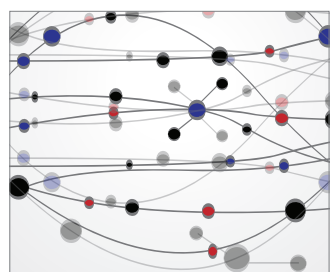

\section{The Scientific} World Journal
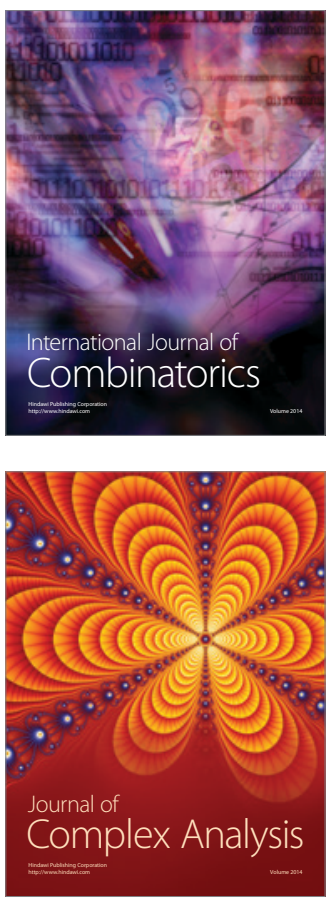

International Journal of

Mathematics and

Mathematical

Sciences
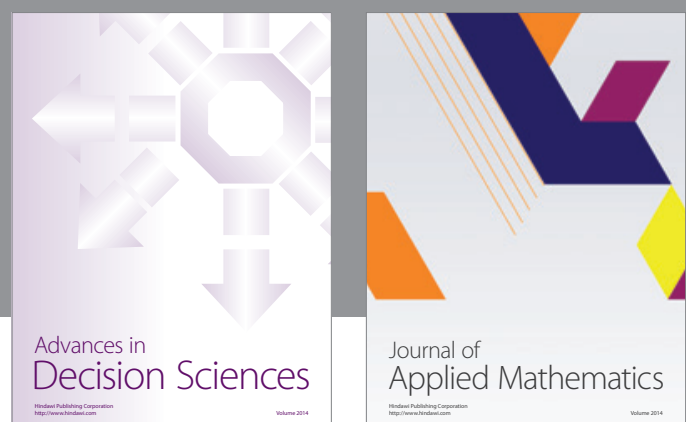

Journal of

Applied Mathematics
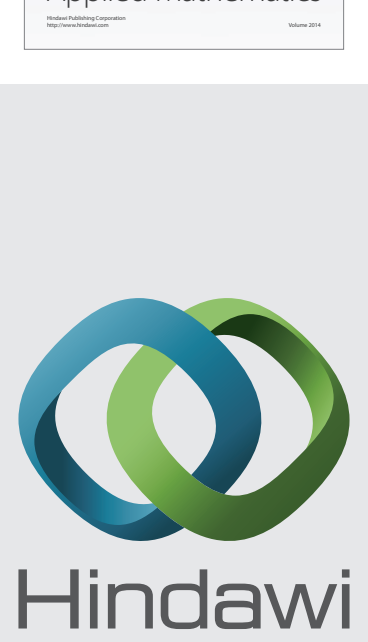

Submit your manuscripts at http://www.hindawi.com
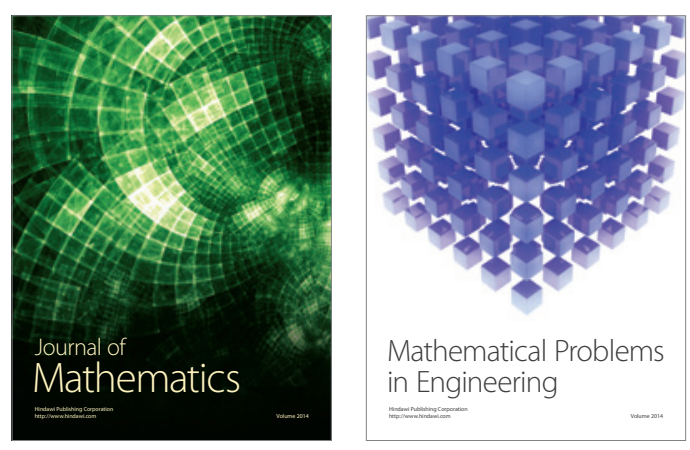

Mathematical Problems in Engineering
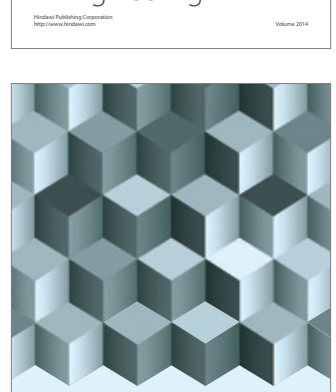

Journal of

Function Spaces
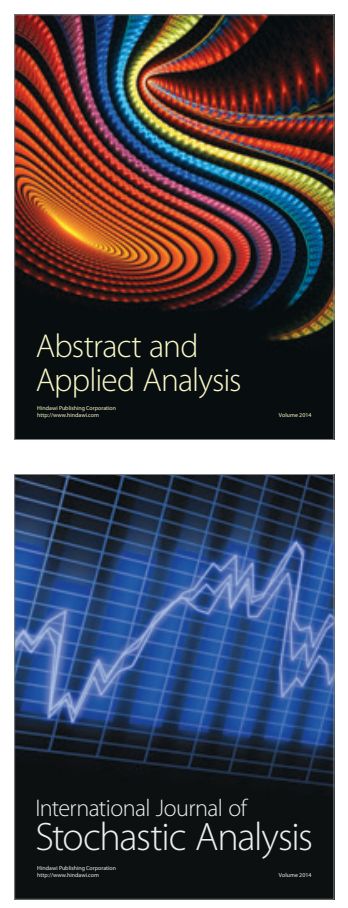

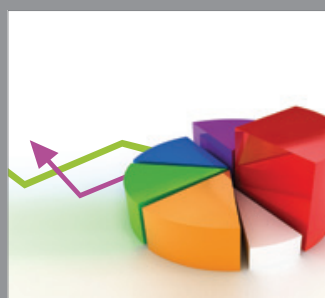

ournal of

Probability and Statistics

Promensencen
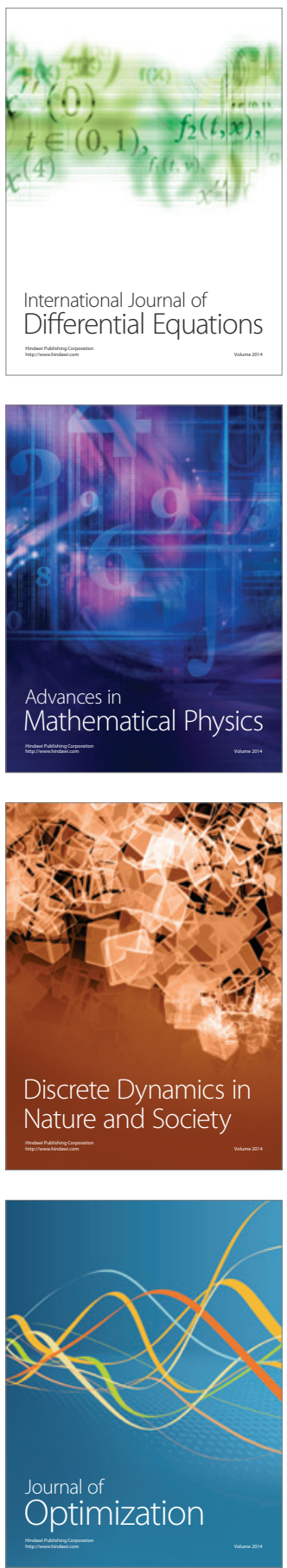